\title{
Posterior Reversible Encephalopathy Syndrome in a Woman With Behcet's Disease: Case Report
}

\author{
Itxaso Azkune-Calle ${ }^{\mathrm{a}}$, Ana Pinedo-Brochado ${ }^{\mathrm{a}}$, Alberto Cabrera-Zubizarreta ${ }^{\mathrm{a}}$, \\ Inigo Vicente-Olabarria ${ }^{\mathrm{a}}$, Juan Carlos Garcia-Monco ${ }^{\mathrm{a}, \mathrm{b}}$
}

\begin{abstract}
Posterior reversible encephalopathy syndrome (PRES) is an entity characterized by the presence of variable neurological symptoms and reversible edema typically affecting the posterior white matter on brain MRI studies. It has been described in association with autoimmune diseases and with the use of chemotherapy and immunosuppressive drugs. We report a case of a 58-year-old woman with Behcet's disease treated with methotrexate, adalimumab and lowdose prednisone who presented with sudden bilateral visual loss, together with radiological findings and clinical course suggesting PRES. PRES may appear associated with Behcet's disease, from whose neurological complications should be differentiated, since management and prognosis differ significantly.
\end{abstract}

Keywords: Posterior reversible encephalopathy syndrome; PRES; Reversible posterior leukoencephalopathy syndrome; RPLS; Hypertensive encephalopathy; Behcet's disease

\section{Introduction}

The posterior reversible encephalopathy syndrome (PRES), first described by Hinchey et al in 1996 [1], is characterized by reversible subcortical edema with variable clinical presentation of neurologic symptoms including headache, confusion, visual disturbances and seizures. The typical neuroimaging findings consist of bilateral, symmetric vasogenic edema involving the white matter of the parietal and occipital lobes. This entity has been described mainly related

Manuscript accepted for publication August 12, 2013

${ }^{\mathrm{a}}$ Department of Neurology and Magnetic Resonance Unit (Osatek), Hospital de Galdakao-Usansolo, Vizcaya, Spain

${ }^{\mathrm{b}}$ Corresponding author: Juan Carlos Garcia-Monco, Department of Neurology, Hospital de Galdakao-Usansolo, 48960 Galdakao, Vizcaya, Spain.Email: hospit05@sarenet.es

doi: http://dx.doi.org/10.14740/jmc1442w to hypertension, renal failure and chemotherapy or immunosuppressive drugs. Hypertension is not always present, particularly in drug-related cases. Although PRES has been reported in the course of several autoimmune disorders, its association to Behcet's disease has not been described so far.

We report the case of a woman diagnosed with Behcet's disease on immunosuppressive therapy, who developed PRES.

\section{Case Report}

A 58-year-old female had been diagnosed with Behcet's disease on the basis of recurrent aphthous oral ulcerations, uveitis and erythema nodosum. She was on methotrexate 2.5 $\mathrm{mg}$ a week, adalimumab every 2 weeks and $2.5 \mathrm{mg}$ of daily prednisone, as well as calcium and vitamin D.

She was admitted for a sudden, bilateral, painless visual loss. Physical examination disclosed complete blindness with light-unresponsive pupils, without any other neurologic or systemic abnormalities. Funduscopy was normal, and ophthalmological evaluation ruled out uveitis or any other ocular problems. Blood chemistry, ESR, CBC and differential were normal. She was afebrile, and her blood pressure was 140/90 mmHg. A cranial CT scan was normal, and a transcranial duplex examination (color mode) showed a less obvious spectrum in the posterior circulation as compared with the anterior circulation. A brain MRI performed a few hours after onset disclosed an abnormal signal on FLAIR sequences over the subcortical white matter of both occipital lobes. MR-angiography showed a less intense representation of the posterior cerebral arteries as compared with the anterior circulation; posterior arteries displayed multiple segmental stenoses (Fig. 1).

Blindness was followed by right-sided neglect and left upper limb ataxia. All the neurological signs resolved spontaneously in $72 \mathrm{~h}$. She remained asymptomatic and with normal blood pressure throughout.

Another brain MRI $72 \mathrm{~h}$ later showed a normalization of the brain parenchyma signal, together with an increase in brain volume of the posterior regions in the perfusion maps, likely reflecting a reactive hyperperfusion (Fig. 1). 


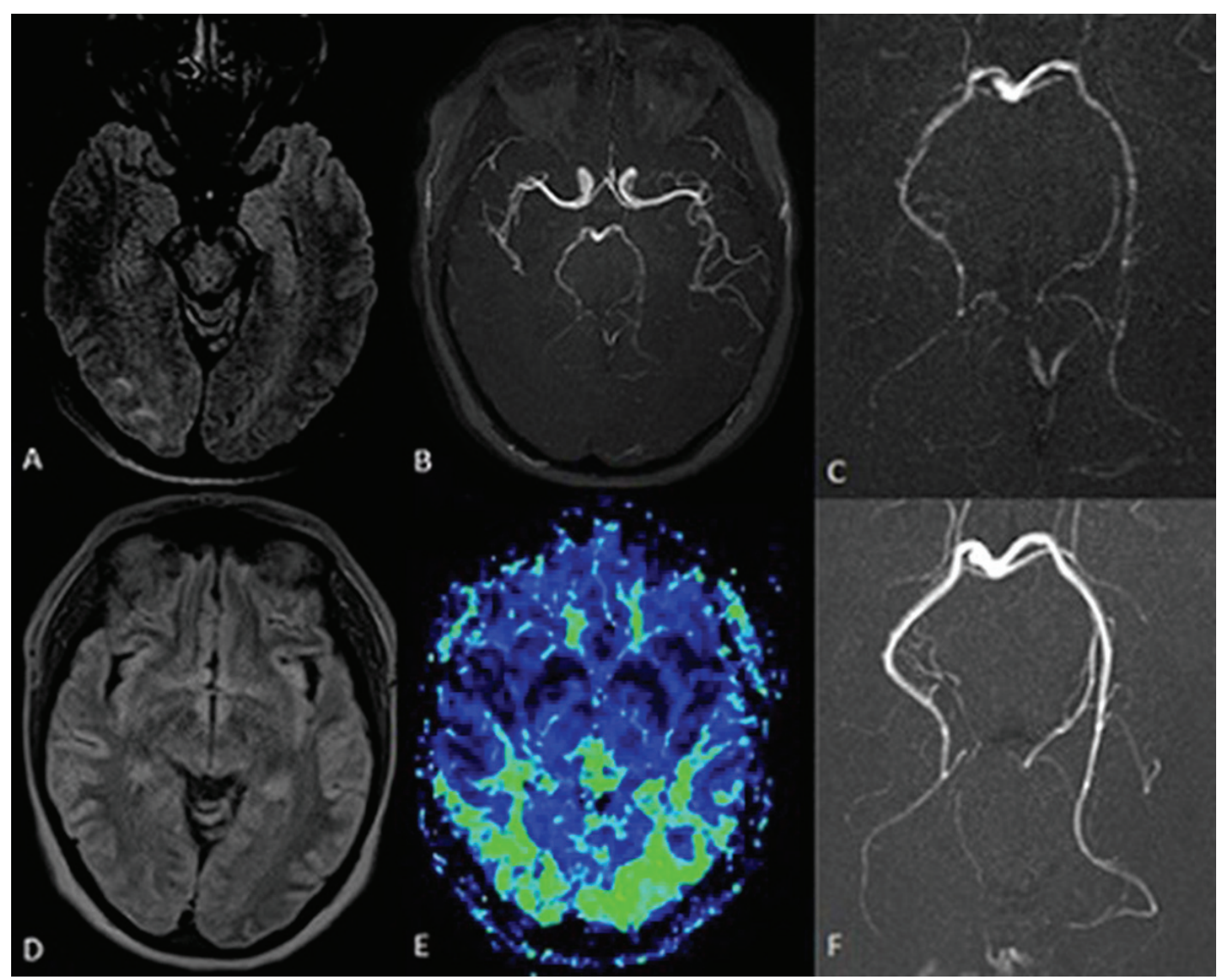

Figure 1. Brain MRI (FLAIR sequences) performed $6 \mathrm{~h}$ after symptoms onset showed edema along the occipital subcortical white matter, more evident on the right side (A). MR angiography $(B, C)$ showed a less intense representation of the posterior cerebral arteries with multiple irregularities in their walls (segmental stenoses). The resolution of the edema is seen on the MRI performed $72 \mathrm{~h}$ later (D), with signs of reactive hyperperfusion of the posterior regions on perfusion maps (E). An angio-MRI performed 4 weeks later showed the resolution of the posterior circulation segmental stenoses $(F)$.

She was discharged, and methotrexate and adalimumab were stopped. An angio-MRI performed 4 weeks later showed resolution of segmental stenoses of the posterior circulation (Fig. 1). She has remained asymptomatic ever since.

\section{Discussion}

PRES is a clinicoradiological entity characterized by headache, confusion, seizures and diverse visual abnormalities in combination with neuroimaging evidence of reversible vasogenic edema predominantly involving the subcortical white matter of parietal and occipital lobes [2].

This patient, with Behcet's disease and immunosuppressive therapy, presented with a painless blindness together with abnormal signal on the occipital lobes and evidence of posterior circulation disturbances on imaging studies. These abnormalities were rapidly reversible, thus suggesting the diagnosis of PRES.

PRES has been described in association to hypertension, eclampsia, organ transplantation, infections, connective tissue disorders, autoimmune disorders, immunosuppressive therapy, chemotherapy, hypercalcemia, uremia, porphyria and HIV patients on antiretroviral treatment $[2,3]$. While PRES has been described in several autoimmune disorders (mainly lupus), we have not found any other case associated to Behcet's disease in the literature.

A number of drugs have been associated to PRES, including cyclosporine, tacrolimus, methotrexate, cyclophosphamide, high-dose steroids and diverse monoclonal antibodies $[2,4]$. This patient was on methotrexate, adalimumab and low-dose prednisone, and their combination could have played a role in PRES development. A sudden increase in blood pressure is the most common trigger for PRES, although it can also occur with normal blood pressure, particularly when it is associated, as in our patient, to chemotherapy or immunosuppressive drugs [5]. A drug-induced endothelial cell damage affecting the brain blood flow autoregulation has been proposed as a pathophysiological mechanism in these patients [2]. 
Neuro-Behcet is the main consideration in the differential diagnosis in this patient. The clinical presentation with a reversible course and the neuroimaging findings would be highly atypical for a small venous inflammatory disease of the brain typical of Behcet's disease [6,7], supporting the diagnosis of PRES. This was the reason why an invasive conventional angiography was not performed. There was no evidence of other neurological complications of Behcet's disease, such as cerebral sinus venous thrombosis, aseptic meningitis or neuropathy.

The diagnosis of reversible cerebral vasoconstriction syndrome (RCVS) should also be considered. This entity is defined by the association of severe headaches (often thunderclap) with or without additional neurological symptoms, and reversible constriction of cerebral arteries resolving spontaneously in 3 months [8]. Our patient had no headache associated with the neurological deficit, and the presence of reversible white matter lesions supported the diagnosis of PRES.

The management of PRES includes blood pressure control and correction of electrolytic abnormalities when present, as well as discontinuation of offending drugs. PRES relapse occurs in approximately $4-8 \%$ of patients, particularly if predisposing factors are not eliminated $[9,10]$.

In summary, the diagnosis of PRES should be borne in mind of physicians caring for patients with systemic disorders including Behcet's disease, since misdiagnosis will likely result in severe neurologic injury or death.

\section{Financial Disclosures}

This manuscript did not receive any financial aid. The authors have no disclosures regarding this manuscript.

\section{Author Contributions}

Drs. Azkune-Calle, Pinedo-Brochado and Garcia-Monco were responsible for the clinical care of the patient, and for writing the manuscript. Dr Vicente-Olavarria and Dr Cabrera-Zubizarreta performed the MRI studies, including their interpretation. They read and commented on the drafts. The final version was read and approved by all the authors.

\section{References}

1. Hinchey J, Chaves C, Appignani B, Breen J, Pao L, Wang A, Pessin MS, et al. A reversible posterior leukoencephalopathy syndrome. N Engl J Med. 1996;334(8):494500 .

2. Bartynski WS. Posterior reversible encephalopathy syndrome, part 2: controversies surrounding pathophysiology of vasogenic edema. AJNR Am J Neuroradiol. 2008;29(6):1043-1049.

3. Guerriero S, Ciraci L, Centoducati T, Pignatelli F, Lamargese V, Salvati A, Dicuonzo F. Bilateral Visual Loss as Presenting Symptom of Posterior Reversible Encephalopathy Syndrome in a Patient with HIV/Tuberculosis Coinfection: A Case Report. Case Rep Ophthalmol Med. 2012;2012:850176.

4. Lazarus M, Amundson S, Belani R. An Association between Bevacizumab and Recurrent Posterior Reversible Encephalopathy Syndrome in a Patient Presenting with Deep Vein Thrombosis: A Case Report and Review of the Literature. Case Rep Oncol Med. 2012;2012:819546.

5. Liman TG, Bohner G, Heuschmann PU, Endres M, Siebert E. The clinical and radiological spectrum of posterior reversible encephalopathy syndrome: the retrospective Berlin PRES study. J Neurol. 2012;259(1):155164.

6. Mohammed RH, Nasef A, Kewan HH, Al Shaar M. Vascular neurobehcet disease: correlation with current disease activity forum and systemic vascular involvement. Clin Rheumatol. 2012;31(7):1033-1040.

7. Ait Ben Haddou EH, Imounan F, Regragui W, Mouti O, Benchakroune N, Abouqal R, Benomar A, et al. Neurological manifestations of Behcet's disease: evaluation of 40 patients treated by cyclophosphamide. Rev Neurol (Paris). 2012;168(4):344-349.

8. Ducros A, Bousser MG. Reversible cerebral vasoconstriction syndrome. Pract Neurol. 2009;9(5):256-267.

9. Sweany JM, Bartynski WS, Boardman JF. "Recurrent" posterior reversible encephalopathy syndrome: report of 3 cases--PRES can strike twice! J Comput Assist Tomogr. 2007;31(1):148-156.

10. Roth C, Ferbert A. The posterior reversible encephalopathy syndrome: what's certain, what's new? Pract Neurol. 2011;11(3):136-144. 